\title{
MATERNAL CONTROL OF HETEROSIS FOR YIELD IN DROSOPHILA MELANOGASTER
}

B. W. BARNES

Department of Genetics, The University, Birmingham, 15

Received 1.ii.68

\section{INTRODUGTION}

IN an investigation into the effect of the environment on a directional selection programme in Drosophila melanogaster Lawrence (1964) suggested that temperature may be able to exert an important influence in two ways. Firstly, on the overall rate of response to selection, and secondly, on the disruption of selection plateaus. The nature of the mechanisms involved in these phenomena have been examined further by a study of the influence of temperature and selection on a population, particular attention being given to their joint effects on fitness (Barnes, 1966).

In a study of this kind a metric for assessing changes in fitness of a population was required. In the literature several suitable metrics have been proposed. However, as fitness must in all cases refer to the ability of an organism to leave offspring, and thereby contribute to the gene pool of subsequent generations, the yield of progeny was used for assessing systematic changes in fitness.

This paper is concerned with an analysis of the genetic system controlling yield, as a knowledge of the components determining the variation will be of obvious importance for any interpretation of changes in the yield of populations subjected to directional selection.

\section{Materials AND methods}

For the purpose of this analysis the parental, $F_{1}, F_{2}$ and backcross generations, including all reciprocals, of the two long inbred lines of Drosophila melanogaster, Oregon, O, and Samarkand, S, were raised in each of three fully randomised blocks set up on the same occasion. The single pair matings were set up in $3 \times 1$ inch vials where the flies were held for two days at $25^{\circ} \mathrm{C}$. before being transferred to the customary one-third pint milk-bottles. Each cross was set up in triplicate within each block and two of the cultures were chosen at random to be scored. The yield of progeny was then scored over a period of five days. All other technical details were those usual for experiments with Drosophila.

\section{Results}

The results as the mean yield over duplicates and blocks are shown in table 1 .

An analysis of variance was performed to test the difference between the generation means and the consistency of the effects over blocks. The results are given in table 2 .

All items in the analysis were tested initially against the pooled duplicates mean square as this was homogeneous over blocks. Only the comparisons 
of particular interest have been shown, all non-significant reciprocal comparisons have been omitted from the table.

The analysis indicates that there is no significant difference between the parents with respect to yield (comparison $\bar{P}_{1} v \bar{P}_{2}$, where $\bar{P}_{1}$ and $\bar{P}_{2}$ are the mean yields of Oregon and Samarkand respectively). No reciprocal differences were detected in the $F_{1}$ or $F_{2}$ generations. With regard to the test for heterosis, a slight modification of the usual procedure was adopted. In most experiments involving the crossing of two inbred lines the two parental lines differ for the character under examination; we would demonstrate

TAble 1

Tield per culture averaged over duplicates and blocks

\begin{tabular}{|c|c|c|c|c|}
\hline \multirow[b]{2}{*}{ 오 } & \\
\hline & $\mathrm{O}$ & $\mathrm{S}$ & $F_{1}(O \times S)$ & $\mathrm{F}_{1}(\mathrm{~S} \times \mathrm{O})$ \\
\hline $\mathrm{O}$ & $120 \cdot 17$ & $159 \cdot 67$ & $135 \cdot 00$ & $100 \cdot 33$ \\
\hline$S$ & $147 \cdot 33$ & $114 \cdot 17$ & $137 \cdot 17$ & $110 \cdot 33$ \\
\hline${ }_{1}(\mathrm{O} \times \mathrm{S})$ & $235 \cdot 83$ & $230 \cdot 33$ & $257 \cdot 83$ & $225 \cdot 50$ \\
\hline$(\mathrm{S} \times \mathrm{O})$ & 283.50 & $247 \cdot 33$ & $253 \cdot 17$ & $220 \cdot 83$ \\
\hline
\end{tabular}

TABLE 2

Analysis of variance of yield data

\begin{tabular}{|c|c|c|}
\hline Item & d.f. & M.S. \\
\hline $\begin{array}{l}\mathbf{P}_{1} v \overline{\mathbf{P}}_{2} \\
\overline{\mathbf{P}}_{v} v \overline{\mathrm{F}}_{1} \\
\overline{\mathrm{F}}_{2} v \overline{\mathrm{F}}_{1}\end{array}$ & $\begin{array}{l}1 \\
1 \\
1\end{array}$ & $\begin{array}{c}108 \cdot 000 \\
7,920 \cdot 667^{*} \\
58,938 \cdot 890^{* * *}\end{array}$ \\
\hline$\overline{\text { B.X. }}\left(\mathbf{O} \times \mathbf{F}_{1}\right) v\left(\mathrm{~F}_{1} \times \mathbf{O}\right)$ & 1 & $120,984 \cdot 000 * * *$ \\
\hline B.X. $\left(\mathrm{S} \times \mathrm{F}_{1}\right) v\left(\mathrm{~F}_{1} \times \mathrm{S}\right)$ & 1 & $79,465 \cdot 042 * * *$ \\
\hline B.X. to $\mathrm{O} v \overline{\mathrm{B} . \mathrm{X}}$. to $\mathrm{S}$ & 1 & $652 \cdot 688$ \\
\hline Blocks & 2 & $564 \cdot 160$ \\
\hline Blocks $\times$ families & 30 & $1,294 \cdot 304$ \\
\hline Duplicates & 48 & $2,328 \cdot 938$ \\
\hline Pooled Error & 78 & $1,930 \cdot 999$ \\
\hline
\end{tabular}

heterosis by showing that the $\mathrm{F}_{1}$ was significantly greater than the better parent. In this case, however, the two parental lines did not differ significantly in yield and therefore heterosis was demonstrated by showing that the $\mathrm{F}_{1}$ yield was significantly greater than that of the mid-parent. The item $\mathrm{P} v \mathrm{~F}_{1}$ was significant indicating heterosis for yield. The comparisons within the backcrosses show that there is a highly significant difference in those crosses in which the $F_{1}$ is used as female parent, compared with those in which the inbred is used, indicating the superiority of the $F_{1}$ as mother as compared with the inbred as mother. Moreover, the $F_{2}$ mean is significantly greater than the $F_{1}$ mean, which is most easily explained as a further manifestation of this maternal effect. There was no significant difference between blocks or any evidence of an interaction between blocks and families.

The analysis, therefore, indicates that both the progeny's own genotype and the maternal genotype are of importance in determining yield.

The results of this experiment also provide a means of assessing whether 
the variation observed in the generation means can be explained on an additive basis, or whether interactions between genes at different loci are important. This can be achieved using a scaling test developed by Cavalli (1952) and used by Jinks $(1954 a, 1955,1956)$ for the detection of nonallelic interactions. The test consists of estimating by weighted least squares the three parameters $[d],[h]$ and $m$, taking as weights the reciprocals of the variances of the generation means. The notation is that of Fisher, Immer and Tedin (1932), where individuals with the genotypes $A A, A a$ and $a a$ have quantitative phenotypes $d a, h a$ and $-d a$ respectively, and where the origin of measurement is the midhomozygote. [ $d]$ and $[h]$ refer to the balance over all loci (Jinks and Morley Jones, 1958). In the example under discussion the backcross generations could not be pooled in the usual way owing to the $\mathrm{F}_{1}$ maternal effect. In view of this an additional parameter, $[f]$, was estimated to take into account the effect due to the genotype of $F_{1}$ mothers. The expectations of the generation means in terms of these parameters, in the absence of non-allelic interactions, are shown in table 3.

A comparison between the observed and expected generation means can then be effected as the sum of squares minimised in the fitting process $\Sigma I_{i}\left(\bar{g}_{i}-\mathrm{E} \bar{g}_{i}\right)^{2}$, where $I_{i}$ is the amount of information about the generation mean and $\tilde{g}_{i}$ is the observed value of the generation mean, is distributed as a $\chi^{2}$ with the degrees of freedom equal to the number of generation means minus the number of parameters estimated.

TABLE 3

Expectations of generation means

$$
\begin{array}{ll}
\mathbf{P}_{1} & =m+[d] \\
\mathbf{P}_{2} & =m-[d] \\
\overline{\mathbf{F}}_{1} & =m+[h] \\
\overline{\mathrm{F}}_{2} & =m+\frac{1}{2}[h]+[f] \\
\mathrm{B}_{2}\left(\mathrm{O} \times \mathrm{F}_{1}\right) & =m+\frac{1}{2}[d]+\frac{1}{2}[h] \\
\mathbf{B}\left(\mathrm{F}_{1} \times \mathrm{O}\right) & =m+\frac{1}{2}[d]+\frac{1}{2}[h]+[f] \\
\mathrm{B}\left(\mathrm{S} \times \mathrm{F}_{1}\right) & =m-\frac{1}{2}[d]+\frac{1}{2}[h] \\
\mathbf{B}\left(\mathrm{F}_{1} \times \mathrm{S}\right) & =m-\frac{1}{2}[d]+\frac{1}{2}[h]+[f]
\end{array}
$$

The values of the parameters estimated together with the results of the scaling test are shown in table 4.

TABLE 4

Scaling test

\begin{tabular}{lcc}
\multicolumn{1}{c}{ Estimate } & $\begin{array}{c}\text { Standard } \\
\text { error }\end{array}$ & Probability \\
$\hat{m}=109 \cdot 4727$ & 10.9667 & $<0.001$ \\
{$[\hat{d}]=5 \cdot 1716$} & 8.9543 & n.s. \\
{$[\hat{h}]=36 \cdot 2089$} & 17.9086 & $0.05-0.02$ \\
{$[\hat{f}]=115 \cdot 8670$} & 8.9543 & $<0.001$ \\
& & \\
$\chi_{4}^{2}=\sum I_{i}\left[\bar{g}_{i}-\mathrm{E} \bar{g}_{i}\right]^{2}=3 \cdot 2221$. & \\
$\mathbf{P}=0 \cdot 70-0.50$.
\end{tabular}

The $x^{2}$ test shows that the model adequately summarises the data. Hence there is no evidence of any non-allelic interactions. The important 
components of the model are dominance in the direction of higher yield, together with a maternal effect associated with the $F_{1}$.

This experiment raises two questions. What is the nature of the superiority of the $F_{1}$ over the parents, and of the $F_{2}$ over the $F_{1}$ ? While the $F_{1}$ heterosis is associated with the genotype of the progeny, and therefore important in the egg, larval and pupal stages, that determining the superiority of the $F_{2}$ is a property both of the progeny's own genotype and the genotype of the $F_{1}$ mother and is presumably mainly important in determining the number of eggs produced and in the hatchability.

A comparison of the magnitudes of the components shows that the heterosis determined by the progeny's own genotype is of minor importance when compared with that determined by the $\mathrm{F}_{1}$ maternal genotype. In the scaling test the parameter $[f]$ was estimated to take into account this maternal effect on an additive scale; we have no indication, therefore, from this source as to the importance of additive, dominance, or interaction components in respect of the mechanism determining the maternal effect. For this reason a further yield experiment was designed to separate the effects of the progeny's own genotype and of the maternal genotype, and more important, to assess the relative magnitudes of the additive, dominance and interaction components of the maternal genotype on yield.

The crosses raised in this experiment are shown in table 5. In the crosses involving a segregating generation, for example $\mathrm{F}_{2} \times \mathrm{O}$, the female parents were chosen at random from the $\mathrm{F}_{2}$ generation, and crossed singly to Oregon males. The yield of progeny over a period of five days was scored from a sample of seven cultures of the crosses having a mother chosen from a nonsegregating generation and from fifteen cultures of the crosses having a mother chosen from a segregating generation. The whole experiment was set up as a single randomised block.

The results of the experiment, as the mean over replicates, together with the model that was tested initially is shown in table 5 .

In the weighted least squares estimation of the components only two

TABLE 5

\begin{tabular}{|c|c|c|c|}
\hline Generation & \multicolumn{2}{|c|}{ Expected mean } & $\begin{array}{c}\text { Observed } \\
\text { mean }\end{array}$ \\
\hline $\mathrm{O} \times \mathrm{O}$ & $m+[d p]$ & {$[d m]$} & $130 \cdot 9421$ \\
\hline$\overline{\mathrm{S} \times \mathrm{S}}$ & $m-[d p]$ & {$[d m]$} & $126 \cdot 4327$ \\
\hline $\mathrm{O} \times \mathrm{S}$ & $+[h p]+$ & {$[d m]$} & $169 \cdot 2857$ \\
\hline $\mathrm{S} \times \mathrm{O}$ & $+[h p]-$ & {$[d m]$} & $161 \cdot 0000$ \\
\hline$\overline{\mathrm{F}_{1} \times \mathrm{O}}$ & $m+\frac{1}{2}[d p]+\frac{1}{2}[h p]$ & $+[h m]$ & $236 \cdot 5714$ \\
\hline$\overline{F_{1} \times S}$ & $m-\frac{1}{2}[d p]+\frac{1}{2}[h p]$ & $+[h m]$ & $256 \cdot 2857$ \\
\hline$\overline{F_{1} \times F_{1}}$ & $+\frac{1}{2}[h p]$ & $+[\mathrm{hm}]$ & $249 \cdot 1429$ \\
\hline$\underline{\mathrm{F}_{2} \times \mathrm{O}}$ & $m+\frac{1}{2}[d p]+\frac{1}{2}[h p]$ & $+\frac{1}{2}[\mathrm{hm}]$ & $235 \cdot 8667$ \\
\hline$\overline{F_{2} \times S}$ & $m-\frac{1}{2}[d p]+\frac{1}{2}[h p]$ & $+\frac{1}{2}[h m]$ & $226 \cdot 3333$ \\
\hline$\overline{F_{2} \times F_{1}}$ & $+\frac{1}{2}[h p]$ & $+\frac{1}{2}[h m]$ & $226 \cdot 7333$ \\
\hline$\overline{F_{2} \times F_{2}}$ & $+\frac{1}{2}[h p]$ & $+\frac{1}{2}[h m]$ & $254 \cdot 2667$ \\
\hline$\overline{B_{1} \times O}$ & $l p]+\frac{1}{4}[h p]$ & $d m]+\frac{1}{2}[h m]$ & $235 \cdot 0667$ \\
\hline$\overline{B_{2} \times S}$ & $m-\frac{3}{4}[d p]+\frac{1}{4}[h p]-$ & {$[d m]+\frac{1}{2}[h m]$} & $250 \cdot 4000$ \\
\hline
\end{tabular}

Where $[d p]=$ additive component of progeny's own genotype, $[h p]=$ dominance component of progeny's own genotype, $[\mathrm{dm}]=$ additive component of maternal genotype, $[\mathrm{hm}]=$ dominance component of maternal genotype. 
weights were used as the variances of the generation means of female parents from non-segregating cultures were homogeneous, as were those from the segregating cultures.

The estimated components, together with the results of the scaling test are given in table 6 .

TABLE 6

Components of generation means

\begin{tabular}{|c|c|c|}
\hline Estimate & $\begin{array}{l}\text { Standard } \\
\text { Error }\end{array}$ & Probability \\
\hline $156 \cdot 1310$ & $10 \cdot 8885$ & $<0.001$ \\
\hline$\widehat{[d p}]-5.9713$ & $10 \cdot 8078$ & n.s. \\
\hline [hp] $19 \cdot 1747$ & $16 \cdot 5315$ & n.s. \\
\hline$[\widehat{d m}] \quad 4.5304$ & $10 \cdot 6486$ & n.s. \\
\hline$[\widehat{h m}] 106.6727$ & $13 \cdot 3256$ & $<0.001$ \\
\hline
\end{tabular}

The scaling test shows there is a highly significant departure of the observed from the expected generation means. The maternal effect of the $F_{1}$ cannot be accounted for on the basis of an additive and dominance component. A more realistic model may be fitted on the basis of the following considerations. The previous experiment showed that the heterosis dependent on the progeny's own genotype can be accounted for principally by a dominance component. There is no evidence showing the importance of non-allelic interactions. We need be concerned, therefore, with interaction components for the maternal genotype only. A model containing [hp], [hm] and a maternal interaction component seems most appropriate.

In the literature there are three principal ways for the specification of nonallelic interactions for the case of two loci, the F $\infty$ metric (Hayman, 1954a), the mixed-metric (Hayman 1954a; Hayman and Mather, 1955), the $\mathrm{F}_{2^{-}}$metric (Hayman, 1955, 1958; Kempthorne, 1957). The relative merits of these methods have been discussed by Van der Veen (1959). The Fळo metric has been used here in specifying the non-allelic interaction components of the maternal effect.

Following the notation of Hayman and Mather (1955), $m$ denotes the origin, $d a, d b, h a, h b$ have the same definitions as given earlier in this section, but extended to the two gene case. For the specification of non-allelic interactions three parameters are required: $i_{a b}$ is the interaction of $d a$ and $d b$ and is termed the homozygote-homozygote interaction; $j_{a / b}$ and $j_{b / a}$ are the homozygote-heterozygote interactions, respectively, of $d a$ and $h b$, and $d b$ and $h a ; l_{a b}$ is the heterozygote-heterozygote interaction of $h a$ and $h b$.

The model fitted to the generation means, together with the results of the scaling test are shown in table 8 . As $[\mathrm{hm}]$ was highly significant while $(\mathrm{dm})$ was non-significant, a model including $[\mathrm{lm}]$ but not $[\mathrm{im}]$ or $(\mathrm{jm})$ seemed most appropriate. The values of the estimated components are given in table 7 .

The non-significant $\chi^{2}$ shows that the model adequately summarises the data. The variation observed in the generation means is ascribable to dominance and interaction components of the maternal genotype. The 
TABLE 7

Components of generation means

\begin{tabular}{|c|c|c|c|}
\hline \multicolumn{2}{|r|}{ Component } & $\begin{array}{l}\text { Standard } \\
\text { error }\end{array}$ & Probabili \\
\hline$\hat{m}$ & 131.5194 & $12 \cdot 1117$ & $<0.001$ \\
\hline & $30 \cdot 7929$ & $16 \cdot 7137$ & $0.10-0.05$ \\
\hline$[\mathrm{hm}]$ & $274 \cdot 7172$ & $38 \cdot 7526$ & $<0 \cdot 001$ \\
\hline$\widehat{l m}$ & $=-174 \cdot 3284$ & $37 \cdot 7511$ & $<0.001$ \\
\hline
\end{tabular}

TABLE 8

Scaling test

\begin{tabular}{|c|c|}
\hline Generatio & Expected value \\
\hline$\overline{\mathrm{O} \times \mathrm{O}}$ & $m$ \\
\hline$\overline{\mathrm{S} \times \mathrm{S}}$ & $m$ \\
\hline $\mathrm{O} \times \mathrm{S}$ & $m+[h p]$ \\
\hline $\mathrm{S} \times \mathrm{O}$ & $m+[h p]$ \\
\hline$\overline{\mathrm{F}_{1} \times \mathrm{O}}$ & $m+\frac{1}{2}[h p]+[h m]+[l m]$ \\
\hline$\overline{\mathrm{F}_{1} \times \mathrm{S}}$ & $m+\frac{1}{2}[h p]+[h m]+[l m]$ \\
\hline$\overline{\mathrm{F}_{1} \times \mathrm{F}_{1}}$ & $m+\frac{1}{2}[h p]+[h m]+[l m]$ \\
\hline $\mathrm{F}_{2} \times \mathrm{O}$ & $m+\frac{1}{2}[h p]+\frac{1}{2}[h m]+\frac{1}{4}[l m]$ \\
\hline$\overline{\mathrm{F}_{2} \times \mathrm{S}}$ & $m+\frac{1}{2}[h p]+\frac{1}{2}[h m]+\frac{1}{4}[l m]$ \\
\hline$\overline{\mathrm{F}_{2} \times \mathrm{F}_{1}}$ & $m+\frac{1}{2}[h p]+\frac{1}{2}[h m]+\frac{1}{4}[l m]$ \\
\hline $\mathrm{F}_{2} \times \mathrm{F}_{2}$ & $m+\frac{1}{2}[h p]+\frac{1}{2}[h m]+\frac{1}{4}[l m]$ \\
\hline$\overline{\mathrm{B}_{1} \times \mathrm{O}}$ & $m+\frac{1}{4}[\mathrm{hp}]+\frac{1}{2}[\mathrm{hm}]+\frac{1}{4}[\mathrm{~lm}]$ \\
\hline$\overline{B_{2} \times S}$ & $m+\frac{1}{4}[h p]+\frac{1}{2}[h m]+\frac{1}{4}[l m]$ \\
\hline
\end{tabular}

dominance effect of the progeny's own genotype is only of borderline significance.

\section{Discussion}

We can turn now to consider the nature of the heterosis observed for yield in the $F_{1}$ and $F_{2}$ generations.

The studies on yield have shown that there are two systems of heterosis that we must be concerned with. The first, the heterosis of the $F_{1}$, is determined by the progeny's own genotype. As egg hatchability is determined principally by the maternal genotype, this heterosis presumably results from the increased larval survival of the $F_{1}$. Bonnier (1961) has demonstrated that the proportion of freshly hatched larvae that reached eclosion was higher in the hybrids between three unrelated wild-type stocks of $D$. melanogaster than in the stocks themselves. The second, the heterosis of the $F_{2}$, is a property of both the progeny's genotype and the maternal genotype, although, as we have seen, the second of these seems to be the most important. This heterosis of the $F_{1}$ mothers most probably results from their increased egg laying capacity. This has been demonstrated to be an important component of heterosis in hybrids of $D$. melanogaster by Bonnier (loc. cit.).

The possible genetic mechanisms determining heterosis have provided material for controversy over the past fifty years. The fundamental point 
at which these theories diverge is whether the extra vigour of the hybrids results directly from heterozygosity per se, or whether it is manifestation of a superior gene content possible in a hybrid where both parental gametes bring in desirable genes. A consideration of this point was discussed at some length by Mather (1955).

The heterozygosity per se interpretation requires the phenomenon of overdominance (Hull, 1945). Jinks (1955), using a method of analysis which allows the discrimination between heterosis arising through overdominance and that arising from interaction between non-allelic genes, analysed certain data published on a wide variety of material and showed that in those cases exhibiting apparent overdominance, non-allelic interaction was also present. In addition, in those cases where the effects of interaction could be reduced in the data, by removing lines showing extreme interactions, the apparent overdominance was reduced.

The importance of the balance of non-allelic interaction components, together with those for additivity and dominance, in determining heterosis has been demonstrated by Jinks and Morley Jones (1958). Heterosis is defined as $\overline{\mathrm{F}}_{1}-\overline{\mathrm{P}}_{0}$, where $\overline{\mathrm{P}}_{0}$ is the better parent. This difference is equal to $|[h]+[l]|-|[d]+[i]|$. Heterosis will result when $[h]$ and $[l]$ take the same sign and $[d]$ and $[i]$ take opposite signs.

In the absence of non-allelic interactions, however, heterosis must be due solely to the additive and dominance components, that is $[h]$ is greater than $[d]$. Two situations may be recognised which can give rise to this effect.

Firstly, it may be a reflection of the gene distribution in the parents (Jinks and Morley, 1958). The parameter $r$ may be taken as a measure of the degree of association or dispersion of the genes in the parental lines, such that when $r=1, \mathrm{P}_{1}$ contains all the dominant increasers, and when $r=-1$ this holds for $\mathrm{P}_{2}$. Under these conditions the definitions of the components of generation means become:

$$
\begin{aligned}
& {[d]=r \Sigma d} \\
& {[h]=\Sigma h}
\end{aligned}
$$

When the dominant increasers are dispersed in the parental lines, $r \rightarrow 0$, the value of $[\hat{d}]$ will be reduced relative to the size of $[\hat{h}]$.

However, if we have association of the genes of greater effect, $|r|=1$, then heterosis is due solely to the fact that $\Sigma h$ is greater than $\Sigma d$. This means that at many loci the heterozygote is superior to either homozygote.

If we now return to the observed results, we have seen that for the heterosis determined by the progeny's own genotype, $[\hat{d}]$ has been estimated as not significantly different from zero, while $[\widehat{h p}]$ was significant. There was no evidence of any non-allelic interactions.

The investigation of the heterosis dependent on the $\mathrm{F}_{1}$ maternal genotype showed that additive effects, $[\widehat{d m}]$, were not significantly different from zero. The principal components were dominance in the direction of high yield, together with an interaction component, analogous to classical duplicate genes. This type of duplicate gene interaction, where $[h]$ and $[l]$ are opposite in sign, cannot, as we have seen earlier, lead to heterosis. We must consider, therefore, the possibility that the significant heterosis shown by the $F_{1}$ and $\mathrm{F}_{2}$ mothers, is the result of overdominance, or of dispersion of dominant increasing alleles in the parental lines. The additive components $[d p]$ and 
$[d m]$ have been estimated as not significantly different from zero, while dominance $[\mathrm{hp}]$ and $[\mathrm{hm}]$ and interaction $[\mathrm{lm}]$ have been the principal components. It is extremely unlikely that there are no additive differences between the lines. This would suggest that as $[d]$ is dependent on $r$, while both $[h]$ and $[l]$ are independent of $r$, i.e., $[d]$ may be a gross underestimate of $\Sigma d$, whereas $[h]$ and $[l]$ are reliable estimates of $\Sigma h$ and $\Sigma l$ respectively, that a system of dispersed genes is involved. The importance of dispersion in the two parental lines has been shown, at least for the genes governing sternopleural chaeta number, by the behaviour of high directional selection lines (Barnes, 1966). This mechanism, rather than a system of genes showing overdominance, is also in agreement with changes of yield observed in random mating lines maintained by double first cousin matings. A system involving overdominance for the major component of fitness, namely the maternal effect, would require that the maximum fitness was achieved only with maximum heterozygosity of the mother. However, random mating lines maintained a high average yield over 40 generations of double first cousin matings (Barnes, 1966). This is not consistent with a heterozygosity per se interpretation.

The final point we may consider concerns the type of genetical architecture displayed by this character. Yield was governed by a system showing strong unidirectional dominance towards high expression, together with highly significantinteractions analogous to duplicate genes of classical genetics. This type of genetical architecture may be related to the forces of natural selection to which the character has been subjected.

Fisher $(1928 a, 1928 b, 1929,1930)$, postulated that where two alleles exist together in a population, natural selection will favour those heterozygotes whose phenotypic expression is changed, by modifiers, towards the phenotype of the homozygote carrying the more favourable allele.

Mather (1960, 1967) and Breese and Mather (1960) have extended this interpretation to include characters governed by polygenic systems. If we consider first characters which have a central optimum, with both extremes at a disadvantage, then we would expect natural selection to be stabilising in action; with stabilising selection dominance might be expected to be incomplete and ambidirectional. However, if we turn now to characters in which high expression in one direction is unconditionally favoured, as with directional selection, then genes pulling in that direction will be expected to show dominance over their alleles. Directional selection will be expected, therefore, to lead to unidirectional dominance.

The evolution of interactions may be expected to follow a similar pattern. This problem has been discussed by Mather $(1960,1967)$. He points out that the conditions necessary for complementary gene interaction must always lead to an increase in the variance of the generation, while the conditions necessary for duplicate gene interaction tend to reduce the variance below the value it takes in the absence of interaction.

Continuing directional selection towards a single optimum phenotype will be expected to favour a reduction of variation. Mather (1967) suggests, therefore, that when natural selection is directional we would expect the evolution of duplicate type interactions. Thus, a system of unidirectional dominance together with duplicate gene interaction will maintain uniformly high fitness in the population.

In Drosophila the total number of progeny produced by a single pair 
mating, as there is no maternal care involved, has been subjected to continuing directional selection. In the present study the observed genetical architecture of yield conforms to that expected, unidirectional dominance for high expression together with marked duplicate type interactions. This type of architecture has been demonstrated also for viability (Breese and Mather, 1960), hatchability, egg production, egg-pupal survival (Keller and Mitchell, 1964) and hatchability (Kearsey, 1966) all in Drosophila, and time to fruiting in Collybia velutipes (Simchen, 1965).

This situation may be contrasted with that observed for characters subjected to stabilising selection. Sternopleural chaeta number in $D$. melanogaster has been demonstrated to be under stabilising selection (Barnes, 1968) and the genetical architecture found is in accordance with this type of selection, i.e. principally additive variation, little evidence of dominance and no interactions. Other characters which have been demonstrated to conform to this genetical architecture are birth weight in rats (Jinks and Broadhurst, 1963), flowering time in Nicotiana rustica (Jinks, 1954), and in Papaver dubium (Lawrence, 1965), number of abdominal chaeta (Breese and Mather, 1957) and body weight (Kearsey and Kojima, 1967) in Drosophila melanogaster.

The results of the present study, taken in conjunction with the accumulating evidence in the literature, suggest that it is possible to infer the type of selection that a character has been subjected to from a consideration of the nature of the genetical architecture displayed by the character.

\section{Summary}

1. The genetic system governing yield in Drosophila melanogaster has been investigated by estimating genetic components of generation means.

2. Two systems of heterosis were found. One system, dependent on the individual's genotype, could be ascribed to unidirectional dominance. The other, dependent on the maternal genotype, showed dominance and duplicate type interaction; overdominance was excluded.

3. This type of genetical architecture has been related to the type of natural selection to which the character has been subjected.

Acknowledgments. - I wish to thank Dr J. S. Gale and Dr M. J. Kearsey for their advice and criticism throughout the course of this work, and Professor J. L. Jinks for his continued interest.

I am indebted to Miss P. Wilkinson for her valuable technical assistance.

This work was carried out during the tenure of an Agricultural Research Council Scholarship.

\section{References}

BARNEs, B. w. 1966. Environment and Selection in Drosophila melanogaster. Ph.D. Thesis, Birmingham.

BARNES, B. W. 1968. Stabilizing selection in Drosophila melanogaster. Heredity (in press).

BONNIER, G. 1961. Experiments on hybrid superiority in Drosophila melanogaster. I. Egg laying capacity and larval survival. Genetics, 46, 9-24.

BREESE, E. L., AND MATHER, K. 1957. The organisation of polygenic activity within a chromosome in Drosophila. I. Hair characters. Heredity, 11, 373-395.

BREESE, E. L., AND MATHER, K. 1960. The organisation of polygenic activity within a chromosome in Drosophila. II. Viability. Heredity, 14, 375-399.

CAVALl, L. L. 1952. An analysis of linkage in quantitative inheritance. Quantitative Inheritance, pp. 135-144. H.M.S.O., London.

FISHER, R. A. 1928a. The possible modification of the response of the wild type to recurrent mutations. Amer. Nat., 62, 115-126. 
FISHER, R. A. 1928b. Two further notes on the origin of dominance. Amer. Nat., 62, 571-574. FISHER, R. A. 1929. The evolution of dominance; reply to Professor Sewall Wright. Amer. Nat., 63, 553-556.

FISHER, R. A. 1930. The Genetical Theory of Natural Selection. Clarendon Press, Oxford. FISHER, R. A., IMMER, F. R., AND TEDIN, O. 1932. The genetical interpretation of statistics of the third degree in the study of quantitative inheritance. Genetics, 17, 107-124.

HAYMAN, B. I. 1954. A mathematical theory of gene action and interaction. Ph.D. Thesis, Univ. of Birmingham Library.

haYMan, в. I. 1955. The description and analysis of gene action and interaction. Cold Spr. Harb. Symp. Quant. Biol., 20, 79-86.

HAYMAN, B. I. 1958. The separation of epistatic from additive and dominance variation in generation means. Heredity, 12, 371-390.

HAYMAN, B. I., AND MATHER, $\mathbf{k}$. 1955. The description of genic interactions in continuous variation. Biometrics, $11,69-82$.

HUll, F. H. 1945. Recurrent selection for specific combining ability in corn. $\mathcal{F}$. Amer. Soc. Agron., 37, 134-145.

JINKs, J. L. 1954. The analysis of continuous variation in a diallel cross of Nicotiana rustica varieties. Genetics, 39, 767-788.

JiNks, J. L. 1955. A survey of the genetical basis of heterosis in a variety of diallel crosses. Heredity, 9, 223-238.

JINks, J. L. 1956. The $\mathrm{F}_{2}$ and Backcross generations from a set of diallel crosses. Heredity, $10,1-30$.

JINKs, J. L., AND BROADHURST, P. L. 1963. Diallel analysis of litter size and body weight in rats. Heredity, 18, 319-336.

JINKS, J. L., AND MORLEY JONES, R. 1958. Estimation of the components of heterosis. Genetics, 43, 223-234.

KEARSEY, M. J., AND koJIMA, к. 1967. The genetic architecture of body weight and egg hatchability in Drosophila melanogaster. Genetics, 56, 23-37.

KELLER, E. C., AND MITCHELL, D. F. 1964. Interchromosomal genotypic interactions in Drosophila. II. An analysis of viability characters. Genetics, 49, 293-307.

Kempthorne, o. 1957. An introduction to Genetical Statistics. John Wiley and Sons, New York.

LAwrence, M. J. 1964. Environment and selection in Drosophila melanogaster. Heredity, 19, 105-124.

LAWRENCE, M. J. 1965. Variation in wild populations of Papaver dubium. I. Variation within populations: Diallel crosses. Heredity, 20, 183-204.

mather, K. 1955. The genetical basis of heterosis. Proc. Roy. Soc., B, 144, 143-150.

MATHER, K. 1960. Evolution in polygenic systems. Int. Colloquium on Evolution and Genetics, pp. 131-152. Rome. Acad. Naz. dei Lincei.

MATHER, K. 1967. Complementary and duplicate gene interactions in Biometrical Genetics. Heredity, 22, 97-103.

smahen, G. 1965. Quantitative inheritance in fungi. Ph.D. Thesis. University of Birmingham Library.

VAN DER VEEN, J. H. 1959. Tests of non-allelic interaction and linkage for quantitative characters in generations derived from two diploid pure lines Genetica, $X X X, 201-232$. 